\title{
Influence of comorbidity on the effect of adjuvant treatment and age in patients with early-stage breast cancer
}

\author{
LH Land ${ }^{*, 1,2}$, SO Dalton ${ }^{3}$, M-B Jensen ${ }^{4}$ and M Ewertz ${ }^{1,2}$ \\ 'Department of Oncology, Odense University Hospital, Sdr. Boulevard 29, Odense C 5000, Denmark; ${ }^{2}$ Institute of Clinical Research, Faculty of Health \\ Sciences, University of Southern Denmark, Winsløwparken 19, 3, Odense C 5000, Denmark; ${ }^{3}$ Unit of Survivorship, The Danish Cancer Society Research \\ Center, Strandboulevarden 49, Copenhagen Ø 2100, Denmark; ${ }^{4}$ The Danish Breast Cancer Cooperative Group, Rigshospitalet 250 I, Blegdamsvej 9, \\ Copenhagen $\varnothing 2100$, Denmark
}

BACKGROUND: Prevalence of comorbidity at breast cancer diagnosis increases with age and is likely to influence the likelihood of receiving treatment according to guidelines. The aim of this study was to examine the effect of breast cancer treatment on mortality, taking age at diagnosis and comorbidity into account.

METHODS: Four nationwide population registries in Denmark: the Danish Civil Registration System, the Danish Breast Cancer Cooperative Group, the Danish National Patient Register, and the Danish Register of Causes of Death provided information on 62591 women diagnosed with early-stage breast cancer, 1990-2008, of whom data on treatment were available for 39943. Comorbidity was measured using the Charlson Comorbidity Index. Adjuvant treatment were categorised as none, chemotherapy, endocrine therapy, and unknown. Multivariable Cox modelling assessed the effect of comorbidity on breast cancer-specific mortality and other cause mortality according to treatment, adjusting for age at diagnosis and other clinical prognostic factors.

RESULTS: The impact of comorbidity on mortality was most pronounced in patients aged 50-79 years. Patients receiving chemotherapy with mild to moderate comorbidity had HR 0.99 (95\% confidence interval (Cl); 0.82-1.19) and I.06 (95\% Cl; 0.77I.46) for breast cancer-specific mortality, respectively, compared with patients without comorbidity.

CONCLUSION: Comorbidity at breast cancer diagnosis is an independent adverse prognostic factor for death after breast cancer. We identified a subgroup of patients with mild to moderate comorbidity receiving chemotherapy who had similar breast cancer mortality as patients with no comorbidity.

British Journal of Cancer (2012) 107, 190I-1907. doi:10.1038/bjc.2012.472 www.bjcancer.com

Published online 18 October 2012

(C) 2012 Cancer Research UK

Keywords: breast cancer; comorbidity; treatment; age; mortality; epidemiology

Evidence on the effect of treatment for early-stage breast cancer derives from randomised clinical trials (RCT) with strict inclusion criteria, such as age, stage of disease, and, in particular, absence of other diseases, which may interfere with the treatment or increase the risk of death (EBCTCG, 2005a, b). These inclusion criteria usually ensure that the patients are relatively young and healthy, that is, free from comorbidity, in order to make the most favourable comparison of the 'experimental' $v s$ the 'standard' treatment (Lewis et al, 2003). Treatment guidelines tend to be based on the results of RCTs. Several studies report that patients aged 65 years or more at diagnosis do not receive treatment according to guidelines just because of their age whereas treatment choice was not influenced by comorbidity (Houterman et al, 2004; Land et al, 2012b). Others have found that patients with comorbidity received less treatment than recommended by guidelines, though the rate of complications to treatment should be similar among those with and without comorbidity (Yancik et al, 2001; Louwman et al, 2005). In Denmark, comorbidity was present in $26 \%$ of breast cancer patients diagnosed 2006-2008 and

\footnotetext{
*Correspondence: Dr LH Land;

E-mail: lotte.holm.land@ouh.regionsyddanmark.dk

Received 28 May 2012; revised 20 September 2012; accepted 21

September 2012; published online 18 October 2012
}

presence of comorbidity increased the risk of dying from breast cancer as well as of other causes (Land et al, 2012a). The aim of this study was to examine the association of early-stage breast cancer and mortality, taking age at diagnosis and comorbidity into account.

\section{MATERIALS AND METHODS}

\section{Data sources and study factors}

Data for the present study were obtained by linking information from four nationwide population registries in Denmark: the Danish Civil Registration System (CRS), the Danish Breast Cancer Cooperative Group (DBCG), the Danish National Patient Register, and the Danish Register of Causes of Death described briefly below.

Civil Registration System was established in 1968 and registers all persons alive and living in Denmark with updated records of vital status and dates of migration. All persons registered in CRS are issued with a unique personal identification number, the CPR number. This CPR number was the key identifier for the record linkages (Pedersen, 2011). Danish Breast Cancer Cooperative Group has performed clinical trials and issued national guidelines for treatment since 1977. Data have been collected on diagnosis, 
histopathology, and surgery for all newly diagnosed early-stage breast cancer patients in Denmark (Møller et al, 2008). For patients enrolled into a clinical trial or who were treated according to the guidelines (denoted 'treatment programme'), information on adjuvant therapy (radiotherapy and medical) is collected. Exclusion criteria were recorded for patients not enrolled into a clinical trial or treatment programme. The National Patient Registry has registered information on all somatic hospital admissions since 1977, and since 1995 data on outpatient and emergency visits, with up to 20 diagnoses per hospitalisation/contact (Lynge et al, 2011). Comorbidity was measured using the Charlson Comorbidity Index (CCI) (Charlson et al, 1987) developed to predict 1-year mortality in medical inpatients, and subsequently validated among breast cancer patients, and against other comorbidity indices (Extermann, 2000b; Klabunde et al, 2007). The CCI provides an overall score for comorbidity based on 19 selected conditions and was categorised as $0,1,2$, and $3+$. Diagnoses other than breast cancer were collected from 10 years before the breast cancer diagnosis and up to the breast cancer diagnosis. Diagnoses of other malignancies in the 2-month period preceding the breast cancer diagnoses were excluded to reduce possible overestimation of malignancies, in the period of diagnostic work-up. The Danish Register of Causes of Death was established in 1875 by the National Board of Health. Since 1970s the register has been fully computerised and includes data of all deaths among Danish residents dying in Denmark (Helweg-Larsen, 2011).

\section{Study population}

The nationwide study population consisted of 62591 women diagnosed with early-stage breast cancer from 1990 through 2008. Complete information on allocated and received treatment was available for 39943 (64\%) of the cohort, while 17338 patients were not enrolled into a treatment programme. This group included patients aged 75 or older at diagnosis, who up to 2002 were not included into the national treatment guidelines, and patients who were ineligible for a treatment programme because they were inoperable, had contraindications for the planned treatment, bilateral breast cancer, prior malignant disease, bone-marrow transplant, metastases at diagnosis, death within 4 weeks of diagnosis, patients' wish, or other causes. For 5310 patients, information was available to allocate the patients into a treatment programme, but no information was recorded on whether the treatment was actually received (Figure 1). Table 1 shows details on tumour characteristics.

\section{Statistical analyses}

Associations between CCI score and age at breast cancer diagnosis, and between CCI score and breast cancer treatment were analysed by $\chi^{2}$ tests. Overall survival (OS) was calculated using the KaplanMeier method, estimating the time from date of surgery until death, irrespective of cause of death, or end of follow-up (31 December 2008). All patients had a surgical intervention: biopsy, lumpectomy, or mastectomy. Cumulative mortality in the presence of competing risks was estimated for deaths from breast cancer, and deaths from other causes, according to CCI score for age at breast cancer diagnosis and treatment, respectively (Grey, 1988; Gooley et al, 1999). The Cox proportional hazards regression model was used (Cox, 1972), to assess the adjusted influence of CCI according to age at breast cancer diagnosis and treatment on breast cancer-specific mortality and other cause mortality, with $\mathrm{CCI}$ score 0 as reference, and time since breast cancer diagnosis as underlying time scale. Adjustments were made for factors associated with prognosis, such as age at breast cancer diagnosis, menopausal status (pre- or postmenopausal according to DBCG criteria (Møller et al, 2008)), tumour size, nodal status, deep fascia invasion, vascular invasion, histological type and grade, hormone
All women diagnosed with breast cancer 1990-2008 in Denmark. $N=62591$

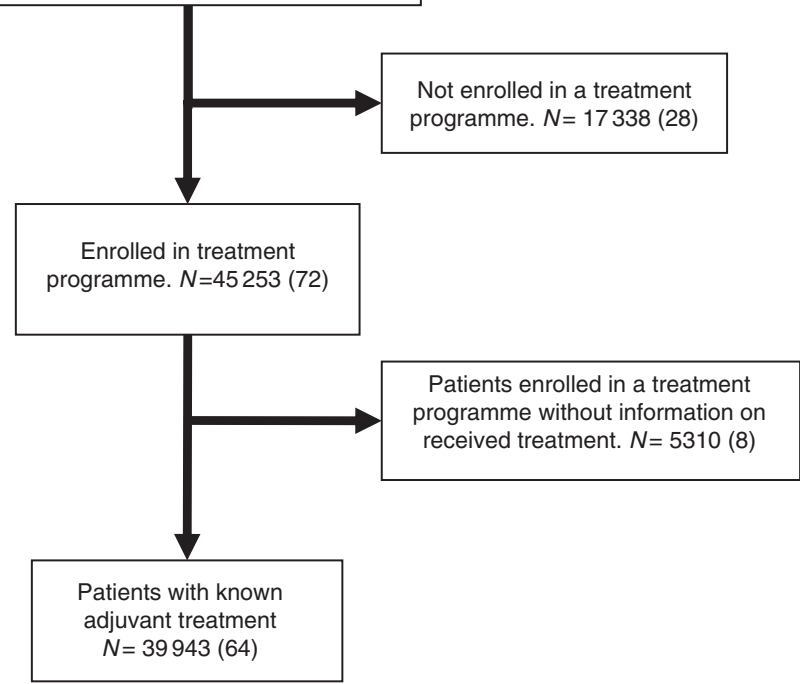

Figure I Diagram showing the study cohort according to received treatment. $N,(\%)$

receptor status, calendar year of diagnosis, and type of surgery and adjuvant treatment. The latter was categorised as: no adjuvant medical treatment $(-)$, chemotherapy (CT) alone or followed by endocrine therapy, and endocrine therapy (E) without chemotherapy. The assumptions of proportional hazards were assessed by plot of Schoenfeld residuals against time and by formal tests (Schoenfeld, 1982). The hazard rates of histological type and grade as well as hormone receptor status were not proportional, and stratified Cox regression was used (Grambsch and Therneau, 1994; Therneau and Grambsch, 2000). To assess possible effect modification, interactions between CCI score and age at breast cancer diagnosis, and between CCI score and received treatment, were investigated in the multivariable Cox model using the Wald test. The division of age at breast cancer diagnosis into three categories, less than 50 years, $50-79$ years, and 80 years or more, derived from multivariable analyses of interactions, of 10 -year age groups where those with similar effects of CCI were grouped together. Tests of statistical hypotheses were done on the two-sided $5 \%$ level of significance. Statistical analyses were performed with the SAS v9.1 statistical software (SAS Institute, Inc., Cary, NC, USA).

\section{RESULTS}

\section{Age at breast cancer diagnosis and comorbidity}

The proportion of breast cancer patients with one or more comorbid conditions at diagnosis increased significantly $(P<0.0001)$ with increasing age at breast cancer diagnosis from $7 \%$ among the 12924 women aged $<50$ years to $21 \%$ in the 43225 aged $50-79$ years, and reaching $40 \%$ among 6442 women 80 years or older (Table 1).

Among patients aged $<50$ years at breast cancer diagnosis, breast cancer-specific mortality was fairly similar for CCI scores $0-2$, but increased for those with CCI $3+$ (Figure 2A). In this age group, breast cancer was the dominant cause of death with few deaths from other causes (Figure 2B). In patients aged 50-79 years at breast cancer diagnosis, mortality increased with CCI score (Figure 2C and D) whereas no such increase was observed in 
Table I Distribution of age at breast cancer diagnosis, adjuvant medical treatment, and tumour characteristics by comorbidity score

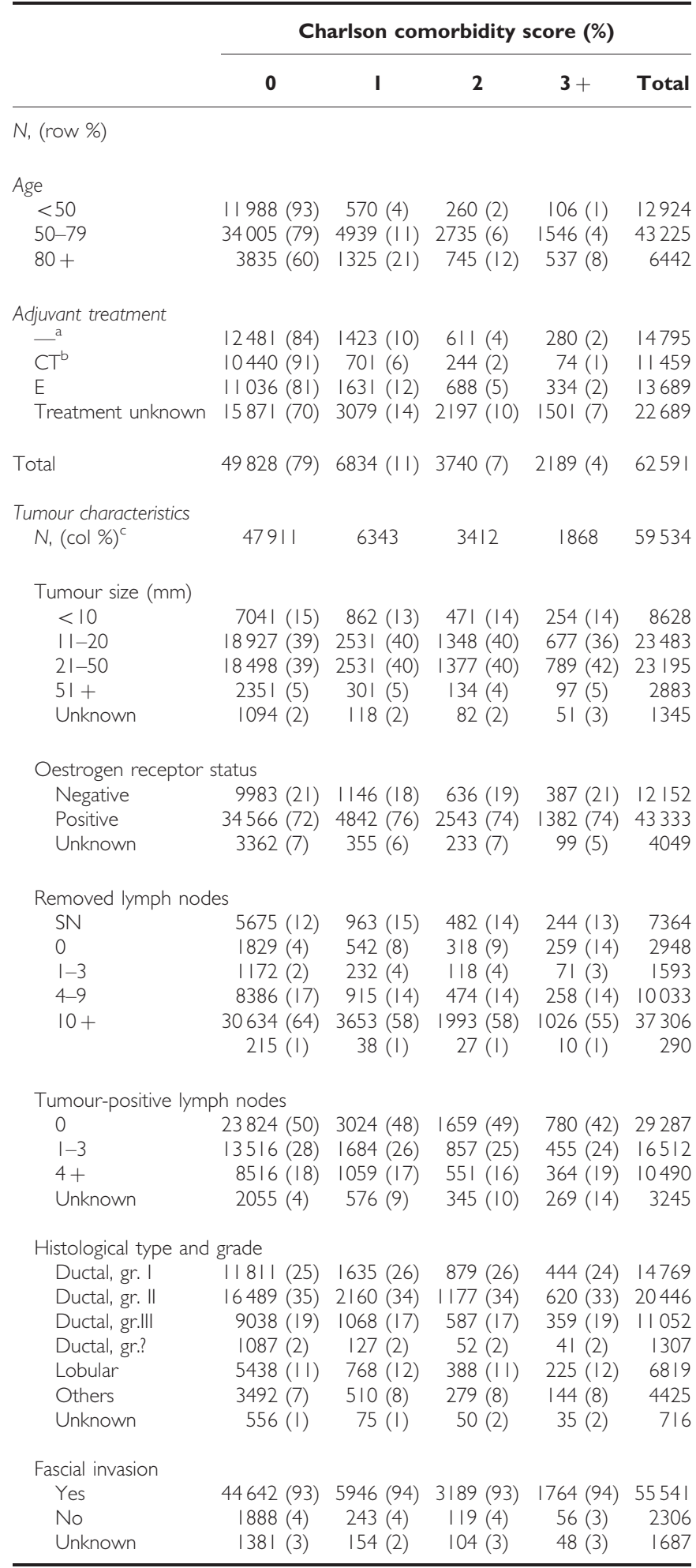

Abbreviations: $\mathrm{CT}=$ chemotherapy; $\mathrm{E}=$ endocrine therapy; gr. = grade; $\mathrm{SN}=$ sentinel node diagnostics. ${ }^{a} \mathrm{No}$ adjuvant medical treatment. ${ }^{\mathrm{b}} \mathrm{CT}$ and $\mathrm{E}$ distributed as follows: CMF (cyclophosphamide, methotrexate and fluorouracil) (4I22), FEC (fluorouracil, epirubicin and cyclophosphamide) (5459), and EC and docetaxel ( 1878$)$. Trastuzumab was given to 587 patients following chemotherapy with FEC or EC and docetaxel. E was almost evenly distributed between tamoxifen alone and tamoxifen followed by aromatase inhibitors (Al), and $\sim 10 \% \mathrm{Al}$ alone. ${ }^{\mathrm{C} T u m o u r}$ characteristics were available on 59534 patients, as 3057 patients were treated with biopsy only. patients aged $80+$ for breast cancer mortality (Figure 2E). While deaths from breast cancer contributed more than deaths from other causes in all age groups, other cause mortality was much higher in patients aged 80 or more years (Figure 2F). A multivariable analysis showed a statistically significant $(P<0.0001)$ interaction between age at breast cancer diagnosis and comorbidity on all cause mortality when adjusting for the effects of known prognostic factors (data not shown).

\section{Treatment and comorbidity}

Figure 3 illustrates OS with 10-year estimates specified among 45253 breast cancer patients enrolled into a treatment programme and 17338 not enrolled. Survival decreased statistically significant with increasing CCI score; except for non-enrolled patients with CCI score 2 who had better survival compared with CCI score 1 . Patients not enrolled in a treatment programme had an OS about half that of patients enrolled. After 15 years, $<25 \%$ of the patients treated outside treatment programme were still alive.

Of the 39943 patients with known adjuvant treatment, 14795 (37\%) belonged to the low-risk group who received no adjuvant treatment, 11459 (29\%) patients received chemotherapy with 4179 of these receiving subsequent endocrine treatment, while 13689 patients received endocrine treatment alone (Table 1). There was a statistically significant interaction between CCI score and treatment on breast cancer-specific mortality, non-breast cancer mortality, and all cause mortality with $P<0.0001$ across all strata for each outcome. Figure 4 shows breast cancer-specific mortality in relation to comorbidity stratified by treatment. Among patients who did not receive adjuvant medical treatment (low-risk patients), those with any comorbidity (CCI $1+$ ) had a higher mortality than those without comorbidity (CCI 0 ) but mortality did not vary much for CCI scores of $1-3+$ (Figure 4A). Patients receiving chemotherapy had similar mortality rates for CCI scores of $0-2$ while those with CCI of $3+$ had a considerably higher mortality (Figure 4B). In the endocrine treatment group, mortality was similar for patients with CCI score land 2 (Figure 4C).

These univariable mortality estimates (Figure 4) were adjusted by multivariable analysis for the effect of other factors known to be associated with breast cancer prognosis and the results are presented in Figure 5. This confirmed that compared with patients with $\mathrm{CCI}=0$, any comorbidity (CCI scores $1-3$ ) was associated with statistically significant increased breast cancer mortality with HRs of 1.56-1.86 among patients with no adjuvant medical treatment. In patients receiving chemotherapy, HRs of $0.99(95 \%$ confidence interval (CI); 0.82-1.19) and HR 1.06 (95\% CI; 0.771.46) for CCIs 1 and 2 were observed compared with women with CCI score 0 while HR for CCI score 3 + was 1.50 (95\% CI; 0.98-2.32). All patients in the endocrine therapy group with comorbidity (CCI $1-3+$ ) had increased HRs ranging from 1.23 to 1.60 (Figure 5A). Breast cancer was the dominant cause of death for patients in all the treatment categories, and as expected, comorbidity highly affected other cause mortality irrespective of treatment group (Figure 5B).

\section{DISCUSSION}

This study confirms that comorbidity at breast cancer diagnosis is an independent adverse prognostic factor for death after breast cancer and that the effect of comorbidity varied by age at diagnosis and by treatment. The relationship between age at breast cancer diagnosis, comorbidity, and mortality is complex as demonstrated by a statistically significant interaction. In patients aged $<50$ years, comorbidity is relatively rare and the predominant cause of death is breast cancer, probably owing to a more unfavourable prognostic profile. Among patients aged 50-79 years, there was a pronounced effect of comorbidity on mortality that increased with 
A

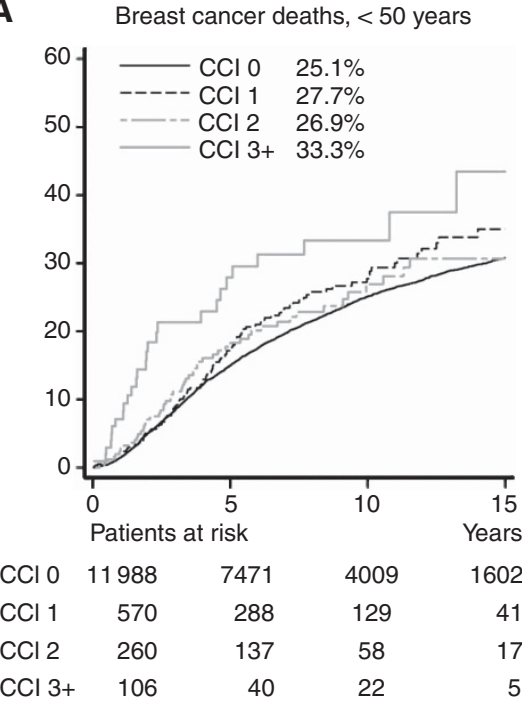

C Breast cancer deaths, $50-79$ years

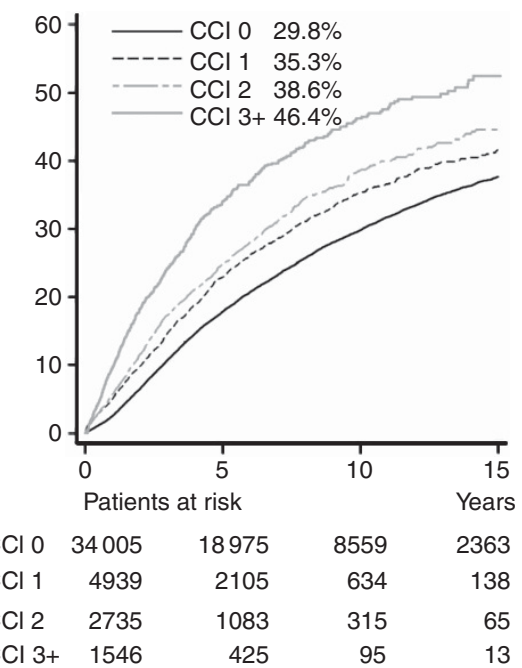

E

E Breast cancer deaths, $80+$ years

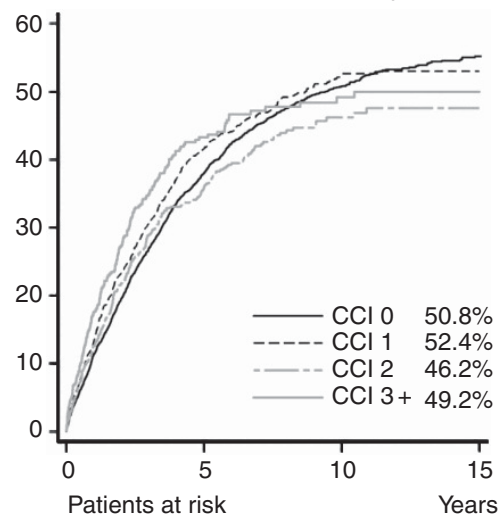

$\begin{array}{lllll}\text { CCI } 0 & 3835 & 1284 & 259 & 23\end{array}$

$\mathrm{CCl} 2 \quad 745 \quad 170 \quad 21$

$\begin{array}{lllll}\mathrm{CCl} 2 & 745 & 170 & 21 & 2\end{array}$

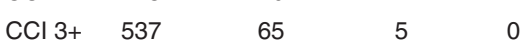

B

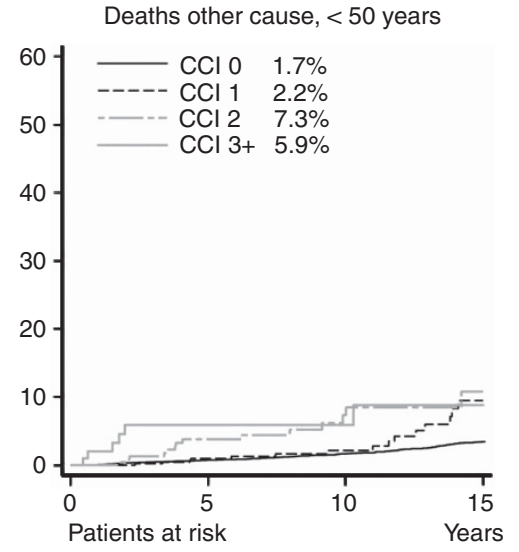

$\begin{array}{lllll}\text { CCI } 0 & 11988 & 7471 & 4009 & 1602\end{array}$

$\begin{array}{lllll}\mathrm{CCl} 1 & 570 & 288 & 129 & 41\end{array}$

$\begin{array}{lllll}\mathrm{CCl} 2 & 260 & 137 & 58 & 17\end{array}$

$\begin{array}{lllll}\mathrm{CCl} 3+ & 106 & 40 & 22 & 5\end{array}$

D Death other cause, $50-79$ years

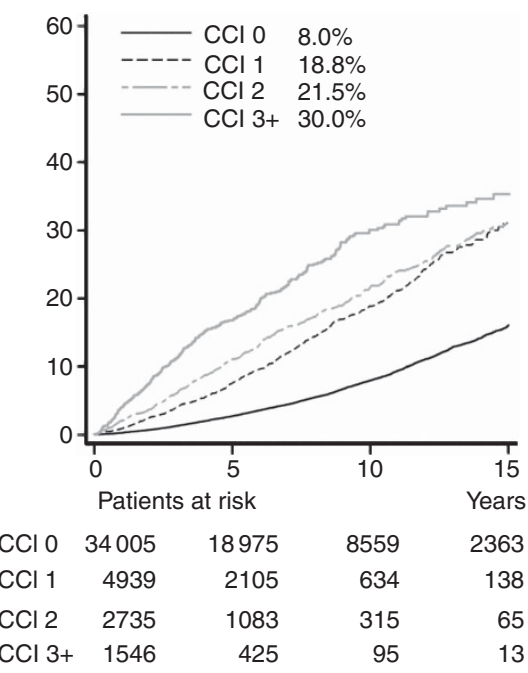

F Deaths other cause, $80+$ yeare

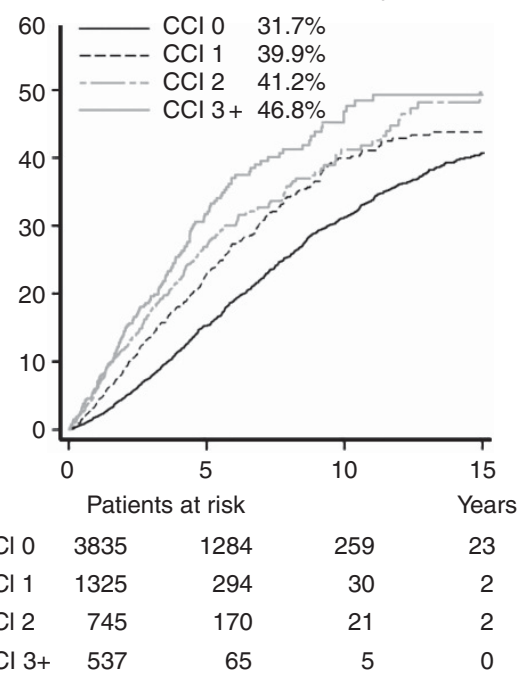

Figure 2 Cumulative mortality (\%) from breast cancer and other causes, according to age at diagnosis and comorbidity score. (A, B) Less than 50 years, (C, D) 50-79 years, and (E, F) $80+$ years. The cumulative mortality at 10 years is listed for each category. 
A

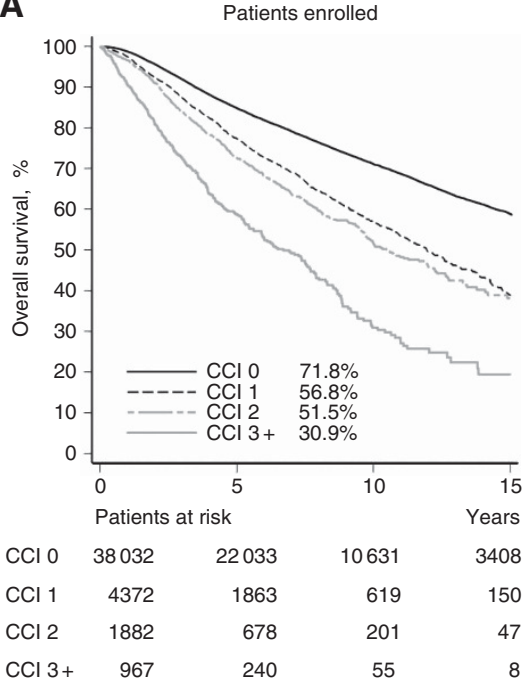

B

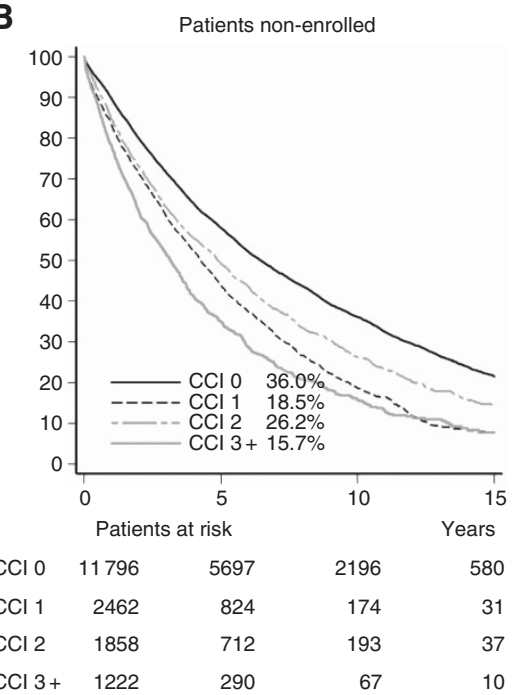

Figure 3 Kaplan-Meier curves showing OS according to comorbidity score for patients (A) enrolled in treatment protocol and (B) non-enrolled in treatment protocol. The OS rate at 10 years is listed for each category.

A

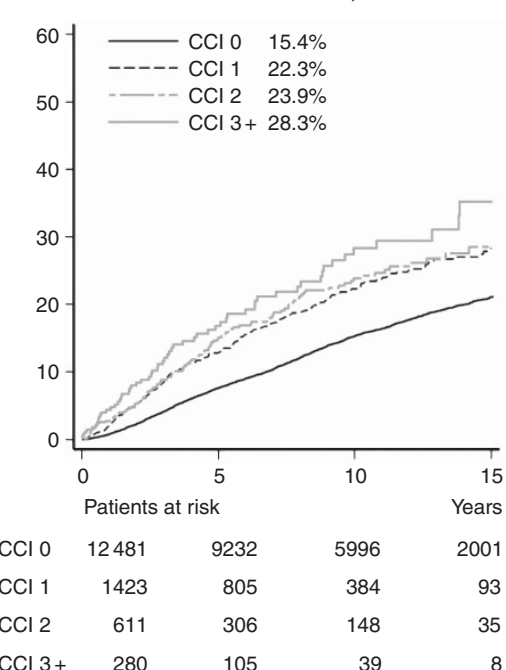

B

B Breast cancer deaths, chemotherapy

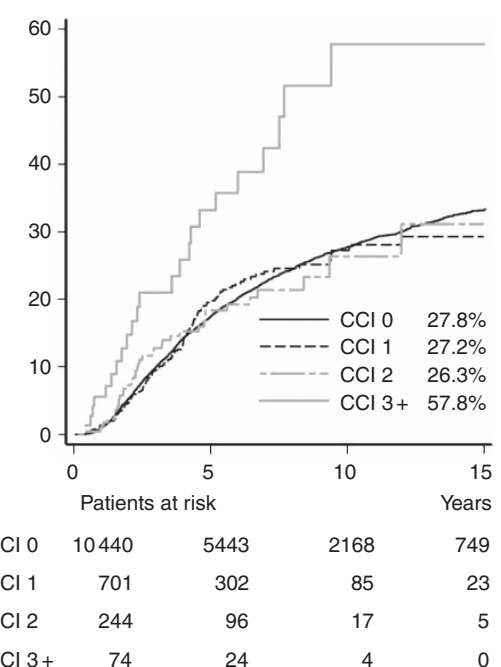

C Breast cancer deaths, endocrine therapy

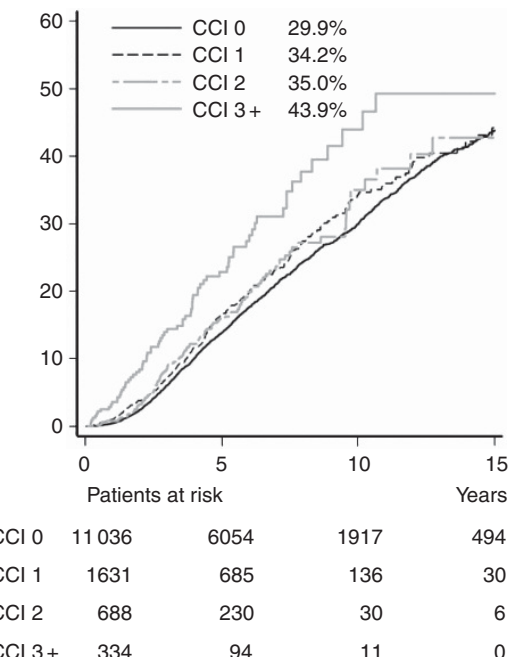

Figure 4 Cumulative mortality from breast cancer for $(\mathbf{A})$ low-risk patients, (B) chemotherapy, and (C) endocrine therapy. The cumulative mortality at 10 years is listed for each category.

increasing CCI score for deaths from breast cancer as well as from other causes. This agrees well with the results from Colzani et al (2011), who demonstrated that the proportion of breast cancer deaths was higher among women younger than 54 years at diagnosis compared with women aged 65-74 years, whereas the proportion of deaths of other causes increased with age at breast cancer diagnosis. In patients aged 80 or more, about $50 \%$ died of breast cancer within 10 years irrespective of comorbidity while the risk of dying from other causes increased with increasing CCI score. It is often difficult to define a single, disease-specific, underlying cause of death in the very old. Discrepancy in causes of death classification between the National Mortality Register and a study committee showed that the proportion of disagreement increased in patients dying at age 85 or older, compared with those dying at age 70 years and younger. Still a high agreement was observed for neoplasms (Alpérovitch et al, 2009).

Age at breast cancer diagnosis may affect treatment decisions, oncologists being more likely to prescribe chemotherapy for a high-risk patient aged 68 years than to an otherwise identical patient aged 73 years (Alistair, 2010). Several studies have reported that elderly cancer patients were undertreated according to national guidelines (Maskarinec et al, 2003; Janssen-Heijnen et al, 2005; Bouchardy et al, 2007; Schonberg et al, 2010; Van Leeuwen et al, 2011). This has also been the case in Denmark where patients aged 75 years or older were not included in the DBCG treatment guidelines up to 2002. Thus, no breast cancer treatment or less than guideline treatment may be a likely explanation for the poorer breast cancer outcome in the oldest age groups.

Previous studies have demonstrated that comorbidity at breast cancer diagnosis increases the risk of dying from breast cancer (Cronin-Fenton et al, 2007; Carlsen et al, 2008; Du et al, 2008; Land et al, 2012a). As with age at breast cancer diagnosis, comorbidity may affect the choice of adjuvant treatment. Patients with severe comorbidity tend to be excluded from RCTs, and the present study demonstrated that patients not enrolled into a treatment programme had twice the breast cancer mortality of those enrolled. Other studies have shown that women who received guideline 
A

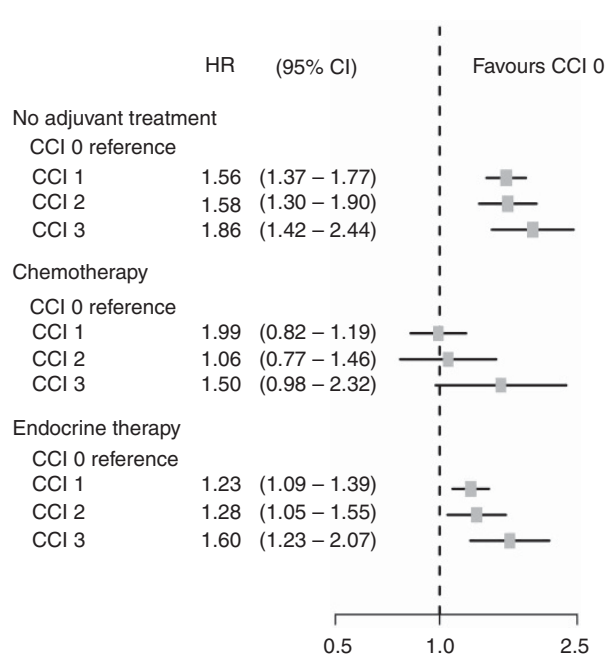

B

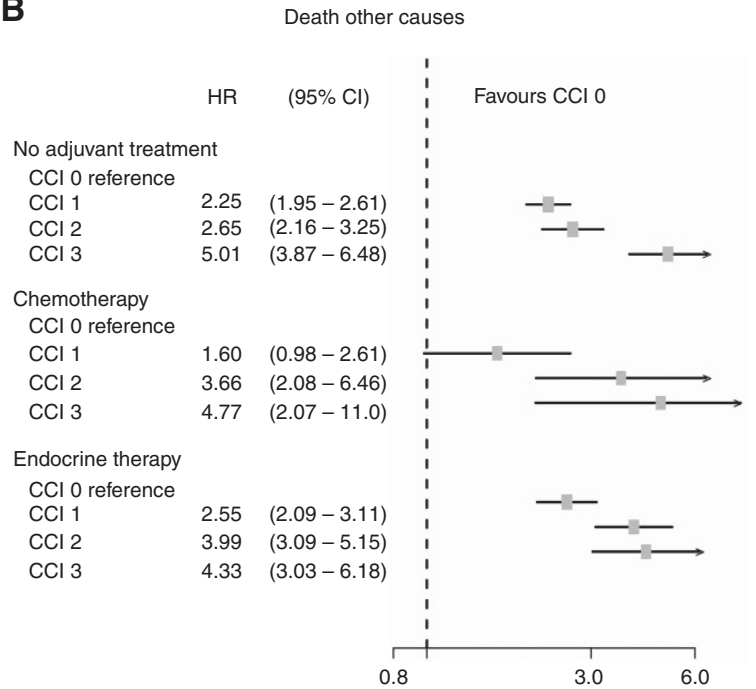

Figure 5 Forest plot illustrating adjusted HR with $95 \% \mathrm{Cl}$ for breast cancer-specific mortality $(\mathbf{A})$ and other cause mortality $(\mathbf{B})$ in relation to CCl and adjuvant treatment, with $\mathrm{CCl} O$ as reference group.

treatment for early-stage breast cancer were statistically significant less likely to die within 5 years than women who did not (Schonberg et al, 2011), and that breast cancer patients with comorbidity had less extensive treatment and a poorer prognosis than patients without comorbidity (Louwman et al, 2005). With the increasing longevity of the female population, oncologists have to face an increasing number of breast cancer patients with comorbidity at diagnosis. It was therefore reassuring to find that among patients for whom chemotherapy was given, the risk of dying from breast cancer was similar for those with mild to moderate comorbidity (CCI scores of 1 and 2) as for those without comorbidity $(\mathrm{CCI}=0)$. We are not aware of other studies reporting similar results and consider this finding new. It should be cautioned, though, that this analysis was based on 945 patients with CCI of 1 or 2 . This group may have included patients whose comorbidity did not involve organ failure or posed contraindication for chemotherapy.

This study has several strengths. It included a large sample size of 62591 women diagnosed with breast cancer in Denmark, during an 18-year time period from 1990-2008, with complete follow-up for vital status and causes of death. Information on received breast cancer treatment was available for 39943 women included in the analysis of treatment and we were able to examine mortality among those for whom treatment was unknown. The information available in the registers is exhaustive, with detailed information on disease characteristic and regarding comorbidity with complete registration of hospitalisations and outpatient visits. We used the CCI, which has been validated in a cohort of breast cancer patients. It is widely used and makes our results comparable with those of others. When mortality is the outcome of interest, the CCI has proven to be a valid and highly appropriate method for measuring comorbidity (Extermann, 2000a; de Groot et al, 2003).

This study has some limitations too. There was no information on received breast cancer treatment for $36 \%$ of the entire cohort and according to CCI, information was unknown for $45 \%$ of patients with $\mathrm{CCI}=1,58 \%$ with $\mathrm{CCI}=2$, and $68 \%$ with $\mathrm{CCI}=3$. This group is likely to differ from those with similar comorbidity but receiving treatment, leading to potential selection bias. The
CCI does not distinguish between severities of the specific conditions, which means that patients with, that is, mild or severe, chronic obstructive pulmonary disease are weighted the same according to the index. Furthermore, the Danish National Patient Registry does not include information on comorbidity that did not require a hospital visit. However, the diseases in the CCI are generally of such serious nature that at some point in the 10-year period preceding breast cancer diagnosis the patient would need a hospital or outpatient contact. Since 1995, the Danish National Patient Registry included emergency and outpatient visits, which could partly explain the increased proportion of comorbidity with calendar time (Land et al, 2012a), though the patients added might be patients with less severe comorbid conditions. Also there are more treatment options for comorbid conditions, which earlier might have been left untreated. Causes of death were ascertained from the Danish Register of Causes of Death and may cause potential misclassification. The validity of the registration of causes of death is threatened by a low and declining autopsy rate. In Denmark at present, the autopsy rate is below $10 \%$, and discontinuity may exist in the reported mortality by uncertain conditions. Furthermore, the quality of the data relies mainly upon the correctness of the physicians' notification (Helweg-Larsen, 2011). Finally, we were not able to adjust for potential confounders, such as overweight, alcohol consumption, and oestrogen supplement, which were not recorded in the registries.

In conclusion, comorbidity at breast cancer diagnosis is an independent adverse prognostic factor for death after breast cancer. The effect of comorbidity on mortality varies with age at diagnosis and with treatment. We identified a subgroup of patients with mild to moderate comorbidity receiving chemotherapy who had similar breast cancer mortality as patients with no comorbidity.

\section{ACKNOWLEDGEMENTS}

The present study was funded by a grant from the Danish Cancer Society.

\section{REFERENCES}

Alistair R (2010) The influences of age and co-morbidities on treatment decisions for patients with HER2-positive early breast cancer. Crit Rev Oncol/Hematol 76(2): 127-132

Alpérovitch A, Bertrand $M$, Jougla $E$, JSb Vidal, Ducimetière $P$, Helmer C, Ritchie K, Pavillon G, Tzourio C (2009) Do we really know the cause of death of the very old? Comparison between official 
mortality statistics and cohort study classification. Eur J Epidemiol 24(11): 669-675

Bouchardy C, Rapiti E, Blagojevic S, Vlastos AT, Vlastos G (2007) Older female cancer patients: importance, causes, and consequences of undertreatment. J Clin Oncol 25(14): 1858-1869

Carlsen K, Høybye MT, Dalton SO, Tjønneland A (2008) Social inequality and incidence of and survival from breast cancer in a population-based study in Denmark, 1994-2003. Eur J Cancer 44(14): 1996-2002

Charlson ME, Pompei P, Ales KL, MacKenzie CR (1987) A new method of classifying prognostic comorbidity in longitudinal studies: development and validation. J Chronic Dis 40(5): 373-383

Colzani E, Liljegren A, Johansson ALV, Adolfsson J, Hellborg H, Hall PFL, Czene K (2011) Prognosis of patients with breast cancer: causes of death and effects of time since diagnosis, age, and tumor characteristics. J Clin Oncol 29(30): 4014-4021

Cox RD (1972) Regression models and life-tables (with discussion). J Roy Stat Soc 34: 187-220

Cronin-Fenton DP, Norgaard M, Jacobsen J, Garne JP, Ewertz M, Lash TL, Sorensen HT (2007) Comorbidity and survival of Danish breast cancer patients from 1995 to 2005. Br J Cancer 96(9): 1462-1468

de Groot V, Beckerman H, Lankhorst GJ, Bouter LM (2003) How to measure comorbidity. a critical review of available methods. J Clin Epidemiol 56(3): 221-229

Du XL, Fang S, Meyer TE (2008) Impact of treatment and socioeconomic status on racial disparities in survival among older women with breast cancer. Am J Clin Oncol 31(2): 125-132

EBCTCG (2005a) Effects of radiotherapy and of differences in the extent of surgery for early breast cancer on local recurrence and 15year survival: an overview of the randomised trials. Lancet 366(9503): 2087-2106

EBCTCG (2005b) Effects of chemotherapy and hormonal therapy for early breast cancer on recurrence and 15-year survival: an overview of the randomised trials. Lancet 365(9472): 1687-1717

Extermann M (2000a) Measuring comorbidity in older cancer patients. Eur $J$ Cancer 36(4): 453-471

Extermann M (2000b) Measurement and impact of comorbidity in older cancer patients. Crit Rev Oncol/Hematol 35(3): 181-200

Gooley TA, Leisenring W, Storer BE (1999) Estimation of failiure probabilities in the presence of competing risks: new representations of old estimators. Statist Med. 18: 695-706

Grambsch PM, Therneau TM (1994) Proportional hazards tests and diagnostics based on weighted residuals. Biometrika 69: 239-241

Gray RJ (1988) A class of K-sample tests for comparing the cumulative incidence of a competing risk. Ann Stat. 16: 1141-1154

Helweg-Larsen K (2011) The Danish register of causes of death. Scand J Public Health 39(7 suppl): 26-29

Houterman S, Janssen-heijnen M, Verheij C, Louwman W, Vreugdenhil G, Sangen MVD, Coebergh J (2004) Comorbidity has negligible impact on treatment and complications but influences survival in breast cancer patients. Br J Cancer 90(12): 2332-2337
Janssen-Heijnen MLG, Houterman S, Lemmens VEPP, Louwman MWJ, Maas HAAM, Coebergh JW (2005) Prognostic impact of increasing age and co-morbidity in cancer patients: a population-based approach. Crit Rev Oncol/Hematol 55(3): 231-240

Klabunde CN, Legler JM, Warren JL, Baldwin LM, Schrag D (2007) A refined comorbidity measurement algorithm for claims-based studies of breast, prostate, colorectal, and lung cancer patients. Ann Epidemiol 17(8): 584-590

Land L, Dalton S, Jensen MB, Ewertz M (2012a) Impact of comorbidity on mortality: a cohort study of 62,591 Danish women diagnosed with early breast cancer, 1990 - 2008. Breast Cancer Res Treat 131(3): 1013-1020

Land LH, Dalton SO, Jørgensen TL, Ewertz M (2012b) Comorbidity and survival after early breast cancer. A review. Crit Rev Oncol/Hematol 81(2): 196-205

Lewis JH, Kilgore ML, Goldman DP, Trimble EL, Kaplan R, Montello MJ, Housman MG, Escarce JJ (2003) Participation of patients 65 years of age or older in cancer clinical trials. J Clin Oncol 21(7): 1383-1389

Louwman WJ, Janssen-Heijnen MLG, Houterman S, Voogd AC, van der Sangen MJC, Nieuwenhuijzen GAP, Coebergh JWW (2005) Less extensive treatment and inferior prognosis for breast cancer patient with comorbidity: a population-based study. Eur J Cancer 41(5): 779-785

Lynge E, Sandegaard JL, Rebolj M (2011) The Danish National Patient Register. Scand J Public Health 39(7 suppl): 30-33

Maskarinec G, Pagano IS, Yamashiro G, Issell BF (2003) Influences of ethnicity, treatment, and comorbidity on breast cancer survival in Hawaii. J Clin Epidemiol 56(7): 678-685

Møller S, Jensen MB, Ejlertsen B, Bjerre KD, Larsen M, Hansen HB, Christiansen P, Mouridsen HT (2008) The clinical database and the treatment guidelines of the Danish Breast Cancer Cooperative Group (DBCG); its 30-years experience and future promise. Acta Oncol (Madr) 47(4): 506-524

Pedersen CBc (2011) The Danish Civil Registration System. Scand J Public Health 39(7 suppl): 22-25

Schoenfeld D (1982) Partial residuals for the proportional hazards regression model. Biometrika 69: 239-241

Schonberg MA, Marcantonio ER, Ngo L, Li D, Silliman RA, McCarthy EP (2011) Causes of death and relative survival of older women after a breast cancer diagnosis. J Clin Oncol 29(12): 1570-1577

Schonberg MA, Marcantonio ER, Silliman RA, Ngo L, McCarthy EP (2010) Breast cancer among the oldest old: tumor characteristics, treatment choices and survival. J Clin Oncol 28(12): 2038-2045

Therneau TM, Grambsch PM (2000) Modeling Survival Data: Extending the Cox Model. Springer: New York

Van Leeuwen BL, Rosenkranz KM, Lei Feng L, Bedrosian I, Hartmann K, Hunt KK, Kuerer HM, Ross M, Singletary SE, Babiera GV (2011) The effect of under-treatment of breast cancer in women 80 years of age and older. Crit Rev Oncol/Hematol 79(3): 315-320

Yancik R, Wesley MN, Ries LAG, Havlik RJ, Edwards BK, Yates JW (2001) Effect of age and comorbidity in postmenopausal breast cancer patients aged 55 years and older. JAMA 285(7): 885-892

This work is published under the standard license to publish agreement. After 12 months the work will become freely available and the license terms will switch to a Creative Commons Attribution-NonCommercial-Share Alike 3.0 Unported License. 